\title{
Le concept de schème syntaxique chez Karl Bühler
}

Par JANETTE FRIEDRICH

Université de Genève

C'est au cours d'une analyse de la pensée verbale que j'ai découvert durant l'année 1907 l'expérienciation (Erlebnis) des schèmes syntaxiques ${ }^{1}$.

\section{Le point de départ : le projet d'une grammaire pure d'un point de vue psychologique}

En 1905, Karl Bühler rejoint l'Institut de psychologie de Würzburg où, depuis quelques années, sont réalisés des travaux expérimentaux sur la psychologie de la pensée sous la direction d'Oswald Külpe ${ }^{2}$. Bühler y commence aussitôt ses propres recherches sur ce sujet, recherches qui aboutissent à sa thèse d'habilitation, publiée en 1907-1908 dans l'Archiv für die gesamte Psychologie. Il est rapporté dans la littérature que c'est Bühler qui a introduit dans les débats de Würzburg les Recherches logiques de Husserl, texte qu'il considérait comme une contribution majeure à la psychologie de la pensée et dont il souhaitait que l'on tienne compte dans les discussions. On trouve, dans ses études sur la pensée, des références explicites aux idées husserliennes, idées qui ont guidé sa propre démarche. Dans son habilitation, Bühler fait également des remarques sur le fonctionnement du langage, même si ce n'est qu'en passant. Il y parle de la "découverte des schèmes syntaxiques », comme l'indique l'épigraphe de ma contribution. Cette découverte me semble intéressante à étudier dans le contexte du présent volume, qui se donne pour but d'interroger l'idée d'une légalité purement grammati-

${ }^{1}$ K. Bühler, Théorie du langage. La fonction représentationnelle, 1934, trad. fr. par D. Samain, Marseille, Agone, 2009, p. 388.

${ }^{2}$ Je remercie Hamid Taieb pour la relecture de mon texte et ses remarques. 
cale telle que proposée par Husserl dans la quatrième Recherche logique. Si Husserl affirme que des principes grammaticaux purement formels conditionnent la possibilité de la signification, il ne met pas seulement en question la prédominance de la logique dans le domaine de la structuration du sens, mais s'oppose aussi à la possibilité de dégager cette légalité d'un examen des différentes langues naturelles. Dès lors, il n'est pas étonnant qu'il se tourne vers les linguistes en les appelant à reconnaître que les lois de la grammaire, comme par exemple celles qui définissent l'intégration correcte des significations partielles dans une signification totale ou les lois qui régissent la relation entre le tout et les parties d'une proposition, sont des lois a priori. Husserl leur suggère de "s'ouvrir à l'évidence que le langage n'a pas seulement des fondements physiologiques, psychologiques et historicoculturels, mais aussi ses fondements aprioriques $»^{1}$.

Il y a là, on le voit, une objection majeure contre l'idée que ces lois pourraient faire l'objet d'une analyse par les sciences empiriques. Dans cette perspective, l'une des questions posées en ouverture du séminaire me semble intéressante à traiter à partir de la conception de Bühler. Elle concerne la pertinence de l'hypothèse d'un fondement apriorique des expressions langagières du point de vue d'un linguiste, et elle a été formulée de la manière suivante : "Quels rapports entretiendrait la grammaire pure avec la légalité spécifiquement linguistique liée à l'expression de ces significations dans telle ou telle langue ?». Peut-on vraiment affirmer que « les catégories de signification et les lois de leur combinaison précèdent tout langage $»^{2}$ ? La réponse que je tente d'esquisser dans ce texte ne va néanmoins pas être celle d'un linguiste, mais celle d'un psychologue. Ma thèse sera qu'on trouve chez Bühler certains arguments qui appuient la thèse d'une grammaire pure, mais qu'ils ne sont ni épistémologiques ni linguistiques: ces arguments sont phénoménologiques, ou psychologiques. Ceci modifiera forcément l'idée d'une grammaire pure. Bühler écrit en 1908, dans le chapitre 3 de son habilitation sur la psychologie de la pensée :

Que sont alors les lois grammaticales? Pour le linguiste, ce qu'on peut abstraire d'une langue donnée ; il les constate, poursuit leur évolution et en a

${ }^{1}$ E. Husserl, Recherches logiques, t. II, partie 2, 1900-1901, trad. fr. par H. Elie, A. Kelkel, R. Schérer, Paris, PUF, 1962, p. 134.

${ }^{2}$ Unité de recherche "Phénoménologies », argumentaire de «Phénoménologie et grammaire », URL : http://www.pheno.ulg.ac.be/colloques/201505-grammaire/fr/. 
terminé avec elles au moment où le psychologue commence son travail. Comment le remplissement de ces lois se fait-il dans le vécu réel ${ }^{1}$ ?

On aurait pu s'attendre à ce que mon argumentation soit développée à partir de la Sprachtheorie, que Bühler publie en 1934 et dans laquelle il élabore sa conception du langage et de la grammaire. Mais Bühler annonce lui-même que c'est dans son habilitation sur la pensée qu'on trouvera l'une des clés pour la compréhension de son œuvre maîtresse. Voici ce qu'il dit dans la préface de la Théorie du langage :

Lorsque, parvenu au point final, je me retourne sur les débuts, il me semble que les bases du système ont été jetées en 1907 avec la découverte des « schèmes syntaxiques » dans la pensée verbale (cf. § 16), et en 1908, lorsque j'ai mis en évidence la fonction de représentation du langage dans mon exposé de synthèse sur les processus de compréhension (III ${ }^{\mathrm{e}}$ congrès de la Société de psychologie) $)^{2}$.

En prenant au sérieux ces remarques rétrospectives, je tâcherai, pour ma part, de me demander si cette dite "découverte» confirme la thèse de la grammaire pure de Husserl ou si elle la met en question. Les pages qui suivent entendent donner d'abord un aperçu de la recherche de Bühler sur la pensée, avant de se concentrer sur leurs principaux résultats et sur les concepts utilisés pour les interpréter.

${ }^{1}$ K. Bühler, « Tatsachen und Probleme zu einer Psychologie der Denkvorgänge: III. Über Gedankenerinnerungen ", Archiv für die gesamte Psychologie, 1908, 12, p. 85.

${ }^{2}$ K. Bühler, Théorie du langage, op.cit., p. 69. 


\section{Les expériences sur la pensée : leurs résultats et leur conceptualisa- $\operatorname{tion}^{1}$}

\subsection{La conscience de la règle}

La question que Bühler pose dans ses expérimentations sur la pensée est la suivante: "Qu'est-ce que nous vivons lorsque nous pensons?» (Was erleben wir, wenn wir denken ?) ${ }^{2}$, «Quels sont les phénomènes que nous éprouvons dans la conscience lorsque nous résolvons des tâches qui mobilisent la pensée ? ». Bühler considère que, jusqu'à présent, la psychologie a identifié la pensée avec la représentation abstraite, ou avec le jugement, ou encore avec l'aperception, tandis que la logique a présenté la pensée comme un acte prédicatif. Il refuse de baser ses recherches sur l'une de ces nombreuses conceptions de la pensée, qu'elles soient psychologiques ou logiques. Son but déclaré est de découvrir le processus psychologique de la pensée et non pas de corroborer une théorie existante. Ainsi, dans ces expérimentations, il propose aux participants d'effectuer des tâches qui demandent l'utilisation de la pensée pour être résolues. Après avoir répondu à la question posée, les participants rapportent ce qu'ils ont vécu durant la résolution des tâches. La méthode utilisée est l'introspection ou, comme le disait Brentano, l'observation mnémonique ${ }^{3}$. Bühler et l'école de Würzburg s'inscrivent bel et bien dans la tradition brentanienne, en prenant au sérieux ce qui était soutenu dans la Psychologie du point de vue empirique, à savoir

\footnotetext{
${ }^{1}$ La psychologie de la pensée de l'école de Würzburg a fait déjà l'objet de deux articles que j'ai publiés en 2008 et 2010, avec chaque fois un thème différent: J. Friedrich, «La psychologie de la pensée de l'école de Würzburg — analyse d'un cas de marginalisation", in M. Kail (éd.), L'homme et la société: Marges et marginalisations dans l'histoire de la psychologie, 2008, $\mathrm{n}^{\circ}$ 167-169, 251-178 et «La pensée comme expérience vécue - l'école de Würzbourg », in Revue de Synthèse, 2010, t. 131, 53-75.

${ }^{2}$ K. Bühler, «Eine Analyse komplizierter Denkvorgänge », in F. Schumann (éd.), Bericht über den II. Kongress für experimentelle Psychologie in Würzburg, Leipzig, Barth, 1907, p. 263.

${ }^{3}$ Il existe un article de Bühler sur ce thème en français : « Remarques sur les problèmes de la psychologie de la pensée ", Archives de psychologie, 1907, 6, p. 376386. Pour une présentation détaillée des recherches de l'école de Würzburg: A. Burloud, La pensée d'après les recherches expérimentales de H.-J. Watt, de Messer et de Bühler, Paris, F. Alcan, 1927.
} 
qu'une utilisation expérimentale de l'introspection «n'est pas exclue du domaine de nos propres phénomènes psychiques $\gg{ }^{1}$.

Dans la suite, Bühler résume les résultats de cette expérimentation et propose des concepts pour les interpréter. Tout d'abord, il constate que l'expérimentation a montré qu'il existe des contenus spécifiques de pensée, des contenus qu'on ne peut pas identifier à des représentations (Vorstellungen) ou à des images mentales. Contre toute tradition associationiste, il affirme que les images sensorielles jouent un rôle minime dans notre expérience de pensée. N'importe quel objet peut être pensé sans auxiliaires intuitifs (Anschauungshilfen), même les couleurs, comme par exemple « cette nuance spéciale de bleu d'un tableau qui est pendu dans ma chambre $»^{2}$. Si le contenu de la pensée n'est pas composé par des représentations mentales, en quoi consiste-t-il ? Bühler répond: "Ce qui ne manque jamais dans la pensée ce sont les pensées (Gedanken) ». Ou, comme il le dit encore : ce qui est attesté dans toutes les expérimentations sur la pensée, c'est l'existence d'un savoir actuel et immédiat (unmittelbares Wissen), d'un savoir entièrement clair, d'un savoir présent ${ }^{3}$. Or, ces deux expressions, celle de «pensées » (Gedanken) et celle de « savoir actuel » ou « présent », sont utilisées comme des équivalents. Loin d'être claire, cette thèse d'un contenu spécifique de la pensée demande des explications. Bühler continue dès lors la restitution des résultats et distingue trois «constituants des vécus de la pensée » (Bestandstücke unserer Denkerlebnisse), qui sont : la conscience de la règle, la conscience des relations et les intentions. Si l'on souhaite comprendre en quoi consiste la spécificité des pensées, il faut donc analyser la manière dont fonctionnent ces constituants. Dans ce qui suit, je me limiterai à la discussion de la conscience de la règle, avec pour but de dégager les traits considérés par Bühler comme communs à tous les processus de pensée. La thèse à démontrer peut être formulée de la façon suivante : les pensées sont constituées par une conscience de la règle, ou, en d'autres termes, par un savoir actuel des règles.

Commençons avec un exemple tiré des procès-verbaux d'expérimentation dans lesquelles les participants cherchent à caractériser leurs actes de pensée par l'introspection (observation mnémonique) et que Bühler cite en faveur de cette thèse :

${ }^{1}$ Fr. Brentano, Psychologie, du point de vue empirique, 1874, trad. fr. par M. de Gandillac, Paris, Vrin, 2008, p. 47.

${ }^{2}$ K. Bühler, «Tatsachen und Probleme zu einer Psychologie der Denkvorgänge: I. Über Gedanken », Archiv für die gesamte Psychologie, 1907, 9, p. 321.

${ }^{3}$ Ibid., p. 360-365. 
D.B42 «Pouvons-nous saisir l'essence de la pensée avec notre pensée »Oui $\left(10^{\prime \prime}\right)$... le savoir qu'une difficulté est suggérée ici qui n'existe tout simplement pas. J'étais conscient de la pensée générale que les phrases dans lesquelles le même concept apparaît d'une certaine manière deux fois ne présentent une difficulté particulière que de manière apparente [...] $]^{1}$.

Cet extrait montre que la personne qui rapporte sa pensée se rend compte que sa réponse a été déterminée par la conscience d'une règle. Une pensée générale est citée comme étant présente durant la solution de la tâche, à savoir : «l'emploi réitéré d'un concept suggère une difficulté qui en réalité n'existe pas ». C'est Ernst Dürr, un collègue de Bühler et l'un des participants à l'expérimentation, qui l'a formulée ainsi rétrospectivement. Cette conscience de la règle était apparemment présente au moment même de penser à la tâche et a mené Dürr à répondre rapidement et sans hésitation par un oui à la question. Pour mieux pouvoir saisir la nature de cette conscience de la règle, Bühler introduit une distinction entre penser à une règle (viser une règle) et la pensée d'une règle (penser dans une règle) :

Je peux aussi simplement viser une règle comme je vise n'importe quel autre objet. La conscience de la règle n'est pas ce penser à une règle, mais la pensée d'une règle ou penser dans une règle. L'objet de la conscience de la règle n'est pas la règle, mais le fait, l'objectivité qu'elle désigne, auquel elle est applicable, duquel elle va peut-être être inférée. Nous pourrions dire, en reprenant une distinction de Husserl, que la conscience de la règle est une pensée dans laquelle certains objets que le logicien désigne comme des lois sont pensées d'une manière adéquate ${ }^{2}$.

Prenons un autre exemple pour clarifier cette distinction. Son choix peut paraître étonnant, car il est emprunté au domaine de la perception. Mais contrairement à ce qui semble être le cas, cet exemple rend particulièrement manifeste le fait que pour Bühler, le fonctionnement de la pensée, du jugement, mais aussi de la perception, bref de toutes les «fonctions psychiques » contient un trait commun. Ainsi, Bühler explique : en regardant les lignes d'une figure mathématique compliquée, au début, je ne sais pas quoi faire avec ces lignes, et puis, d'un coup, je le sais, je le saisis. Qu'est-ce que je sais, qu'est-ce que j'ai saisi ? demande Bühler. «Apparemment, le sens de la figure », et il poursuit : " ce sens est en tout cas quelque chose d'idéel,

${ }^{1}$ Ibid., p. 337.

${ }^{2}$ Ibid., p. 339-340. 
dans beaucoup de cas rien d'autre que sa loi » ${ }^{1}$, même si cette loi n'est pas sa loi exacte, complète et précise, mais une règle brute de la constitution de la figure. Cette saisie de la règle, dont Bühler parle ici, n'équivaut pas à penser à une règle. Je ne pense pas à une règle puis perçois la figure. Je pense dans la règle, ce qui veut dire qu'en regardant la figure, une règle de la constitution de la figure m'est présente, ce qui fait qu'une Gestalt est perçue.

Cette conscience de la règle ne doit pas être confondue avec la référence à une règle existante sous forme d'un savoir que, donc avec la référence à une proposition. Bien au contraire, dans la conscience de la règle, la proposition apparait comme quelque chose d'immédiatement connu. Bühler nous rappelle que quelque chose de semblable se passe quand je « comprends » d'un coup la construction d'une machine ou le plan d'un bâtiment. Comme l'a souligné plus tard Gilbert Ryle en proposant une distinction entre le knowing how et le knowing that, le savoir comment, par exemple la capacité de faire de bonnes plaisanteries, n'est pas à confondre avec la connaissance des règles, car ce n'est pas le fait de penser aux canons esthétiques qui explique cette capacité. Dans sa fameuse argumentation contre «le fantôme dans la machine », Ryle souligne que «la pratique de l'humour n'est pas un sous-produit de la théorie de l'humour. Les canons du goût esthétique, du tact dans les manières et de la technique de l'invention peuvent rester informulés sans gêner l'exercice intelligent de ces capacités $\rangle^{2}$. Il en va de même pour la conscience de la règle, où la règle peut rester non-remarquée et non-exprimée tout en étant présente durant la résolution de la tâche. Ainsi, la distinction bühlerienne entre penser à la règle et penser dans la règle semble thématiser le même phénomène.

\footnotetext{
${ }^{1}$ Ibid., p. 341.

${ }^{2}$ G. Ryle, La notion d'esprit. Pour une critique des concepts mentaux, 1951, trad. fr. par S. Stern-Gillet, Paris, Payot, 1978, p. 99-100.
} 


\subsection{Une clé de lecture : les références à l'école de Brentano ${ }^{1}$}

Mais il existe encore une autre « clé de lecture » pour clarifier la psychologie de la pensée de Bühler. Dans la citation ci-dessus, Bühler fait clairement référence à un débat célèbre, celui concernant le psychologisme. C'est comme s'il cherchait à signaler qu'il était bien conscient du danger dénoncé par Frege, Husserl et bien d'autres, et qu'il tentera de l'éviter. En utilisant l'appareillage conceptuel développé par les membres de l'école de Brentano pour interpréter les résultats de ses expérimentations, Bühler insiste sur le fait que la conscience dans la règle ou de la règle, conscience qui conduit à percevoir des Gestalten, à pouvoir comprendre des aphorismes, n'est pas une règle de la pensée considérée comme acte psychologique d'une personne. Il ne s'agit aucunement d'une règle de la pensée de nature empirique que le psychologue pourrait découvrir dans l'analyse de la pensée de la personne. Rappelons ce que Bühler écrit à ce propos : «L'objet de la conscience de la règle n'est pas la règle, mais le fait, l'objectivité qu'elle désigne, auquel elle est applicable ». Il s'oppose donc à l'idée que la règle qui est présente du point de vue de la première personne, du point de vue de la personne qui pense ou, comme on pourrait dire aussi, du point de vue de la conscience phénoménale, serait existante exclusivement sur ce plan-là. La pensée d'une règle indique toujours une objectivité, elle nous renseigne dès lors sur l'existence de la règle à un autre niveau que celui des fonctions psychiques. Cet autre niveau a été désigné entre autre par Stumpf, Husserl et Twardowski comme celui des "formations». Parmi les formations, les brentaniens comptaient les lois idéelles, les vérités logiques, les connaissances géométriques, les valeurs morales, les critères de beauté, etc., bref des types d'objets bien spécifiques, à savoir des objets idéaux, ayant une validité objective. Il a été dit à leur propos qu'il serait impossible de les inférer à partir des fonctions psychiques.

Si on utilise cette clé de lecture, «je pense dans la règle » veut donc dire qu'une règle est attestable, par exemple, dans la perception d'une figure. Toutefois, cette règle attestée n'est pas à confondre avec le produit d'une

${ }^{1}$ Sur la relation entre Bühler et Stumpf, voir D. Fisette, « Phénomènes sensibles et fonctions psychiques : Karl Bühler et le programme de Stumpf », in L. Cesalli \& J. Friedrich (éds.), Anton Marty \& Karl Bühler, Between Mind and Language, Zwischen Denken und Sprache, Entre pensée et langage, Basel, Schwabe, 2014, p. 103-140. Voir aussi G. Fréchette, «Der Ausgangsgegenstand der Psychologie. Bühler und die Schule Brentanos », in J. Friedrich \& Fr. Stadler (éds.), Karl Bühlers Krise der Psychologie (1927). Positionen, Bezüge und Kontroversen im Wien der 1920er/30er Jahre, Wien [u.a.], Springer, (sous presse). 
pensée individuelle. Bien au contraire, elle possède un caractère objectif et est indépendante du fonctionnement de la pensée en question. C'est ce que Bühler cherche à exprimer quand il propose cette étonnante correspondance : « La conscience de la règle est une pensée dans laquelle certains objets que le logicien désigne comme des lois sont pensés d'une manière adéquate $»$. De fait, au début de son habilitation, on trouve une référence fort intéressante à Husserl. Bühler affirme que Husserl aurait instauré une méthode transcendantale qui suppose un remplissement des lois logiques grâce aux phénomènes psychiques, une méthode qui suppose un lien entre les deux, et qui force à fondamentalement réviser notre conception du psychique, en renonçant à l'idée d'une non-correspondance ${ }^{1}$. Dans un autre article, intitulé «Zur Kritik der Denkexperimente » et publié en 1909, Bühler réagit à un certain nombre d'objections qui ont été formulées après la publication de son habilitation. Il s'y réfère à un autre élève de Brentano, Carl Stumpf, et affirme que ce que lui-même avait discuté dans son habilitation à l'aide du terme de «Regelbewusstsein » (conscience de la règle) aurait été thématisé par Stumpf par un autre terme, celui de « forme »: «Stumpf considère, lui aussi, ces mêmes contenus comme un groupe spécifique de "formations de fonctions psychiques", pour lesquels il propose le nom de "formes", et il les place au même niveau que les relations $\rangle^{2}$.

Bühler cite le texte de Stumpf intitulé "Phénomènes et fonctions psychiques $»^{3}$ et trouve notamment dans le paragraphe «Digression sur les formations des fonctions psychiques » des appuis utiles à sa propre argumentation. L'expression «formations de fonctions psychiques» est d'ailleurs utilisée à plusieurs reprises dans son habilitation ${ }^{4}$. Il y a notamment deux affirmations qui sont importantes à retenir selon lui. D'une part, il y a l'idée qu'une formation (un état de choses, un "Inbegriff» ou un ensemble) est ce que les brentaniens appellent un «corrélat de fonctions psychiques». Rappelons-le, une formation n'est pas une fonction psychique en tant que telle. Ainsi, Stumpf le souligne en précisant que l'Inbegriff, pour Husserl,

${ }^{1}$ K. Bühler, «Tatsachen und Probleme zu einer Psychologie der Denkvorgänge: I. Über Gedanken », op. cit., p. 298-299.

${ }^{2}$ K. Bühler, «Zur Kritik der Denkexperimente », Zeitschrift für Psychologie, 1909, 51, p. 116.

${ }^{3}$ Bühler publie en 1908 un compte-rendu sur ce texte : K. Bühler, «Besprechung von: C. Stumpf, Erscheinungen und psychische Funktionen », Archiv für die gesamte Psychologie, 1908, vol. 11, p. 1-5.

${ }^{4}$ Cf. aussi K. Bühler, «Nachtrag. Antwort auf die von W. Wundt erhobenen Einwände gegen die Methode der Selbstbeobachtung an experimentell erzeugten Erlebnissen », Archiv für die gesamte Psychologie, 1908, 12, p. 113. 
n'est ni la fonction synthétique, ni le matériau synthétisé, réuni, mais «le corrélat nécessaire de la fonction synthétique ». Il poursuit en affirmant que chacune des fonctions psychiques aurait un tel corrélat. D'autre part, Bühler tente de nous persuader de son hypothèse de correspondance, prenant également appui sur Stumpf qui, tout en insistant sur la différence entre fonctions psychiques et formations, admet néanmoins un contenu commun : «Dans la mesure où les formations sont aussi des contenus des fonctions psychiques, elles ont toutes un caractère objectif et ne conservent rien dans leur concept de l'acte individuel du moment ${ }^{1}$. D'où l'insistance de Stumpf sur le fait qu'il faut clairement distinguer les fonctions psychiques, qui constituent l'objet de la psychologie, des formations (Gebilde), qui, elles, sont analysées par la philosophie. Dans son texte «De la classification des sciences », publié la même année, soit en 1906, il insiste : "La nécessité de distinguer les formations tant des phénomènes que des fonctions, de la manière la plus manifeste dans le domaine de la logique et celui de la théorie de la connaissance, doit s'imposer à la philosophie présente $»^{2}$. Nous l'avons vu, Bühler est pleinement d'accord. Il est lui aussi fermement opposé à toute forme de psychologisme. Néanmoins, il insiste en même temps sur le fait que cette position n'est aucunement en contradiction avec l'hypothèse de la correspondance entre fonctions psychiques et formations.

D'après sa vision, les formations « apparaissent » deux fois : une fois en tant que contenu objectif, donc en tant que corrélat existant indépendamment de l'acte psychique de pensée, et une deuxième fois comme la pensée d'un sujet, comme un acte psychique individuel dans lequel ce même contenu est attestable. Nous l'avons vu ci-dessous, la conscience de la règle n'est pas un vécu subjectif, mais un contenu objectif, un corrélat, qui peut être indiqué soit rétrospectivement par la personne même qui pensait, soit par un observateur extérieur, par un interprète, comme ayant été présent dans l'acte de penser. Le cas de Dürr l'a montré : ce qu'il énonce n'est pas une loi de l'acte de penser vécu, mais la connaissance d'une loi énoncée après coup comme ce qui avait déterminé la réponse. Un contenu objectif (une règle) est présent dans l'acte psychique de penser, sans pour autant qu'il trouve son

${ }^{1}$ C. Stumpf, «Phénomènes et fonctions psychiques », 1906, éd. et trad. fr. par D. Fisette, in C. Stumpf, Renaissance de la philosophie. Quatre articles, Paris, Vrin, 2006, p.158.

${ }^{2}$ C. Stumpf, « De la classification des sciences », 1906, éd. et trad. fr. par D. Fisette, in C. Stumpf, Renaissance de la philosophie. Quatre articles, op.cit., p. 198 ; voir aussi p. 174-175 et p. 199, 201. 
explication dans cet acte même. Or, l'explication consiste dans le fait que ce contenu est le corrélat objectif (la formation) de l'acte de penser. Autrement dit, quelque chose est déterminant dans ce processus, sans que cela soit nécessairement conscient pour le sujet. On le voit bien, il n'est pas si évident d'échapper à l'impression que l'expression de "conscience de la règle » prête quelque peu à confusion. Car ce n'est pas la prise de conscience d'une règle par la personne qui pense qui est visé par Bühler, mais la pensée dans une règle, une pensée de la règle, une pensée qui est conforme à la règle telle qu'elle existe en tant que formation sans être nécessairement consciente pour l'individu. Cette hypothèse de la correspondance, à laquelle Bühler tient, est néanmoins une sorte de rouage tournant à vide. Comme c'est le cas pour n'importe quelle correspondance entre des choses qui sont en même temps considérées comme fortement différentes, nous y rencontrons une difficulté insurmontable. Car si le contenu des fonctions psychiques n'est explicable qu'à partir des formations, s'il n'est rien d'autre qu'une répétition sur le plan individuel du contenu objectif des formations, pourquoi faire encore de la psychologie? Pourquoi analyser le fonctionnement psychologique si son contenu n'est pas explicable par une telle recherche ? Pour échapper à cette difficulté, Bühler s'intéresse encore, dans son habilitation, à une autre observation faite durant ses expérimentations.

\subsection{Penser, c'est présenter quelque chose indirectement}

Une grande partie du dispositif d'expérimentation sur la pensée se basait sur l'utilisation de la mémoire. Pour résoudre les tâches proposées, les participants ont dû se souvenir des mots ou des expressions qui ont été lus au début de l'expérience. Dans l'analyse des résultats, Bühler montre que les pensées ayant lieu au moment de la mémorisation n'interviennent pas en tant que telles dans le processus actuel de pensée. C'est la raison pour laquelle à la question «qu'est-ce qu'est un vécu de souvenir ? », Bühler répond «nous trouvons en tout cas en lui une influence actuelle qu'un vécu antérieur gagne sur un vécu présent $»^{1}$. La relation entre la pensée antérieure et la pensée actuelle est une relation dans le présent et non une relation entre un événement passé et un événement présent. On se réfère à la pensée antérieure

${ }^{1}$ K. Bühler, «Tatsachen und Probleme zu einer Psychologie der Denkvorgänge: III.
Über Gedankenerinnerungen », Archiv für die gesamte Psychologie, 1908, 12, p. 61. 
sans la revivre, dit Bühler ${ }^{1}$. Dans cette perspective, il convient de noter la distinction de Bühler entre la "réactualisation d'une pensée antérieure » (Wiederaktuellwerden der früheren Gedanken) et la «remémoration d'une pensée antérieure » (ein sich an die früheren Gedanken erinnern). C'est la première qui intervient dans les réponses des participants : «Les participants à l'expérience se réfèrent effectivement à l'objet antérieur, mais d'une manière indirecte comme à "l'objet qui" se trouve dans une relation logique déterminée avec l'objet présent $»^{2}$. La présence de la pensée antérieure dans la pensée actuelle transforme dès lors la pensée antérieure, puisque ce qui est convoqué de la dernière est ce qui est visé (demandé) par la pensée actuelle. Les participants ne se rappellent pas des mots pour ensuite pouvoir résoudre avec eux la tâche, mais ces mots retenus antérieurement (le souvenir) s'imposent dans la résolution de la tâche en fonction d'elle, donc indirectement. Pour Bühler, il s'agit ici d'un exemple prototypique du mécanisme de la présentation indirecte.

D'autres exemples de présentation indirecte sont cités et discutés dans l'habilitation de Bühler. Regardons encore un autre extrait des procèsverbaux et écoutons les commentaires que Bühler en donne. Cette fois-ci, c'est la réponse d'Oswald Külpe, directeur de l'Institut de psychologie de Würzburg, mais aussi l'un des participants à l'expérimentation, qui est citée ci-dessous :

K.B42 'Pouvons-nous saisir l'essence de la pensée par la pensée ?' - Oui (6") — La question m'a d'abord semblé bizarre ; j'ai pensé qu'il s'agissait

${ }^{1}$ Dans les Recherche philosophiques, Wittgenstein discute le même phénomène en affirmant que pouvoir continuer la suite des nombres entiers naturels dans le système décimal ne se fond pas sur l'expérience qui a été faite dans le passé d'une telle application : «Tu veux donc dire que tu sais comment appliquer la loi de la suite, tout à fait indépendamment du souvenir que tu as de ses applications effectives à des nombres déterminés. » (L. Wittgenstein, Recherches philosophiques, trad. fr. par É. Rigal (dir.), Paris, Gallimard, 2004, p. 98).

${ }^{2}$ K. Bühler, «Tatsachen und Probleme zu einer Psychologie der Denkvorgänge. III. Über Gedankenerinnerung », op. cit., p. 69. Dans la première partie de son habilitation, dans le paragraphe "La constitution de la pensée », Bühler affirme déjà que ce sur quoi porte la pensée peut être déterminé selon deux manières: directement et indirectement. "Je peux penser à quelque chose soit comme "ceci", soit comme " la chose qui... ". Dans le dernier cas, je n'y pense qu'en tant qu'elle est cela, c'est-à-dire qu'elle présente ces particularités, alors que dans le premier cas, je pense à quelque chose dont je sais éventuellement par ailleurs qu'il présente ces particularités. » (K. Bühler, "Tatsachen und Probleme zu einer Psychologie der Denkvorgänge I. Über Gedanken », op. cit., p. 359, voir aussi p. 360). 
d'une question-piège. Puis le reproche de Hegel à Kant m'est brusquement venu à l'esprit, et alors j'ai dit résolument oui. La pensée que j'ai eue du reproche de Hegel avait un contenu assez riche, sur le coup j'ai su exactement de quoi il s'agissait, mais je n'ai rien verbalisé, je n'ai pas eu de représentation mentale, seul le mot de Hegel a résonné après coup en moi (du point de vue acoustique et moteur) ${ }^{1}$.

Külpe rapporte l'impression qu'il embrassait, par l'acte de pensée, un contenu extrêmement riche. Le savoir entier de toute une période de l'histoire de la philosophie lui était apparemment présent. Pourtant, il est impossible de se référer en si peu de temps à un savoir d'une telle ampleur et de le passer en revue, rétorque Bühler. Il ne fait aucun doute que ce qui a été évoqué par Külpe n'a pas pu être saisi dans sa totalité, même si ses paroles semblent suggérer une telle conclusion. Voici donc l'interprétation que Bühler propose. L'exemple cité montrerait que ce qui se passe dans le vécu de la pensée ne peut pas être réduit à des contenus ${ }^{2}$. Car dans le cas cité, il faut supposer qu'il y avait d'autre chose qui a permis de répondre si rapidement à la question posée. Et cet autre chose a été, selon Bühler, un ordre psychique des choses. Il explique que dans cet ordre psychique des choses, il existe une place marquée et vide pour n'importe quel objet et penser le monde consisterait donc dans le fait de se référer à cette place. Ce qui est conscient au moment où nous pensons, ne sont pas des contenus, mais quelque chose comme un ordre dans lequel ce sur quoi porte la pensée, tout ce à quoi nous pouvons penser comme à quelque chose de déterminé, a une place et est ainsi connu. On y retrouve en effet l'idée de la présentation indirecte, puisque le contenu visé est pensé indirectement, à travers la place que la chose a dans un ordre donné. L'ordre ne représente pas le contenu, mais donne des points d'orientation à travers lesquels le contenu peut être pensé. Regardons comment Bühler introduit cette notion d'ordre psychique des choses pour la première fois dans son habilitation :

nous pouvons dire: les quiddités dans les actes de savoir immédiat de quelque chose sont des déterminations de places au sein d'un ordre conscient. Nous ne connaissons pas encore cet ordre ou ces ordres [...] et il faut donc ajouter que c'est «comme si», c'est pour le moment une description symbolique. Mais nous pouvons déjà déduire certaines phrases

\footnotetext{
${ }^{1}$ K. Bühler, «Tatsachen und Probleme zu einer Psychologie der Denkvorgänge: I. Über Gedanken », op. cit., p. 305.

${ }^{2}$ Voir K. Bühler, «Tatsachen und Probleme zu einer Psychologie der Denkvorgänge: I. Über Gedanken », op. cit., p. 348-349.
} 
(thèses) quant à cet ordre hypothétique. S'il y a une telle chose dans la conscience, cela devrait être quelque chose de très courant, quelque chose de si évident qu'il nous échappe jusqu'au moment où on nous questionne sur lui. Par ailleurs, il doit y avoir de la place pour tout ce à quoi nous pouvons penser comme à quelque chose de déterminé sans contenus conscients remplissants ; et c'est tout ce qui peut devenir en général pour nous un objet. Appelons-le l'ordre psychique des choses $[\ldots]^{1}$.

Ces ordres hypothétiques vont de soi, ils sont évidents et les personnes qui les utilisent dans leurs pensées ne se rendent pas compte de leur présence. Nous pouvons ici constater le même fonctionnement que pour la conscience de la règle : la prise de conscience (de la règle ou de l'ordre) ne précède pas la pensée, mais cela n'empêche pas, bien au contraire, que la règle ou l'ordre soit présent dans la pensée. Ces ordres jouent un rôle fondamental, car ils permettent de penser à une chose comme à quelque chose de déterminé sans que nous nous référions à un contenu de conscience, sans qu'un remplissement ait lieu ${ }^{2}$. Bühler évoque ici la possibilité de confondre cet ordre avec un signe, un signe qui tient la place de ce qui est visé, mais il s'oppose aussitôt à cette idée. La quiddité des objets est bel et bien pensée et pas seulement signalée, puisque la place dans l'ordre psychique des choses indique des relations quant aux autres choses, et ces relations ne peuvent exister qu'entre des choses. L'ordre ne représente pas l'objet, mais oriente la pensée de l'objet, en d'autres termes il le présente comme celui qui..., comme un objet déterminé, étant caractérisé par le lien qu'il a avec d'autres objets du même ordre. La saisie de ce lien est donc ce qui fait connaitre l'objet. En résumant, on pourrait dire que : dans la compréhension de Bühler, penser veut dire être orienté, être guidé par un ordre psychologique des choses à travers lequel une certaine chose est connue. Il semble donc peu étonnant que Bühler, dans sa

\footnotetext{
${ }^{1}$ Karl Bühler, « Tatsachen und Probleme zu einer Psychologie der Denkvorgänge: I. Über Gedanken, op. cit., p. 357-358. Dans la Krise der Psychologie, Bühler revient sur le rôle joué par cet ordre pour la pensée mais également pour la perception : «Cette manière d'englober, de saisir par la pensée ce qui est présent sur le plan perceptif grâce à la détermination de sa place dans un ordre est un cas spécifique de la détermination structurelle dans le domaine phénoménal. » (K. Bühler, Krise der Psychologie, 1927, Frankfurt/M., Berlin, Wien, Ullstein, 1978, p. 117). Voir aussi Charlotte Bühler, « Über Gedankenentstehung. Experimentelle Untersuchungen zur Denkpsychologie », Leipzig, Barth, 1918.

${ }^{2}$ Bühler compare la notion d'ordre psychique des choses à la notion husserlienne d'acte purement signitif (rein signitive Akte) (comme visée à vide d'un objet), une comparaison qui me semble faire sens. K. Bühler, «Tatsachen und Probleme zu einer Psychologie der Denkvorgänge: I. Über Gedanken », op. cit., p. 359.
}

\section{1}


Psychologie de la pensée, se réfère aux mathématiques, plus précisément aux fonctions mathématiques, dont il loue les performances :

On peut, par exemple, penser clairement et d'une façon sûre la décroissance en fonction du carré de la distance, sans déterminer les termes entre lesquels la distance existe et ce qui décroît ; c'est réellement la fonction même qui est pensée et clairement présente à la conscience, tandis que ce à quoi elle se rapporte peut se réduire à quelque chose d'indéterminé, qui n'est pensé que comme un point de comparaison ou comme le substitut vide d'un contenu de relation ${ }^{1}$.

La discussion menée jusqu'ici a présenté la pensée comme un processus psychique basé sur des présentations, telles que l'ordre psychique ou les fonctions mathématiques, afin de saisir un contenu qui n'est pas accessible en tant que tel. Cette position de Bühler met gravement à mal l'idée que la pensée serait une manipulation spécifique de contenus, qu'elle serait composée par des représentations mentales ou encore qu'on pourrait découvrir dans les recherches psychologiques sur la pensée des contenus correspondants à la pensée. Selon Bühler, la pensée s'effectue toujours indirectement, donc par l'utilisation de moyens d'auxiliaires. Pourquoi en vat-il ainsi ? Il me semble que la réponse est intimement liée au problème de la nature psychique de la pensée. La pensée se fait indirectement car il est psychologiquement impossible de saisir uno intuitu dans la pensée tout ce qui est pensé ${ }^{2}$. Les objets (objets abstraits) avec lesquels on opère dans la pensée ont une telle nature qu'ils ne peuvent être pensés que par une «présentation», donc indirectement. Bühler indique même la source pour

${ }^{1}$ K. Bühler, «Tatsachen und Probleme zu einer Psychologie der Denkvorgänge: I. Über Gedanken », op. cit., p. 340-341, cité par A. Burloud, La pensée d'après les recherches expérimentales de H.-J. Watt, de Messer et de Bühler, op. cit., p. 131.

${ }^{2}$ Christiane Chauviré, dans ses études sur la philosophie mathématique de Peirce, montre que ce dernier discutait les diagrammes comme des quasi-réalités, comme une entité seconde « qui lui (au mathématicien) résiste et agit sur lui », et qu'ils sont nécessaires à la pensée mathématique car «les propriétés du diagramme que le mathématicien va découvrir ne sont déjà là que parce qu'il les y a mises, peut-être sans en avoir eu clairement conscience, en construisant ou en faisant évoluer le diagramme, et, s'il va devoir les découvrir, ce n'est pas parce qu'elles sont cachées, mais parce qu'il lui est psychologiquement impossible, souligne Peirce, de les saisir toutes uno intuitu. » (Chr. Chauviré, «Perception visuelle et mathématiques chez Peirce et Wittgenstein », in J. Bouveresse et J.-J. Rosat (éds.), Philosophies de la perception. Phénoménologie, grammaire et sciences cognitives, Paris, Odile Jacob, 2003, p. 210-211).

\section{2}


cette manière d'aborder le problème de la pensée, en se référant à Kant et à son idée du schématisme de la raison. C'est en fait l'idée d'un contenu intuitif des concepts qui y est en jeu. Nous le savons tous, il est possible de se faire une image du nombre 5 ou même du nombre 10, en désignant par exemple 5 ou 10 points. Mais comment faire si on veut se représenter (imaginer) un objet aussi abstrait que le nombre 100 ou 1000 ? Un tel objet, on ne peut que le penser, et Kant fait justement référence à un « moyen » qui est nécessaire pour cela :

$\mathrm{Au}$ contraire, quand je ne fais que penser à un nombre en général, qui peut être cinq ou cent, cette pensée est la représentation d'une méthode pour représenter une multitude (par exemple, mille) dans une image, conformément à un certain concept, plutôt que cette image même, qu'il me serait difficile, dans le dernier cas, de parcourir des yeux et de comparer au concept. Or, c'est cette représentation d'un procédé général de l'imagination pour procurer à un concept son image que j'appelle le schème de ce concept ${ }^{1}$.

Le problème auquel l'interprète est ici confronté est la question de savoir ce que peut bien vouloir dire "procédé général de l'imagination». Qu'est-ce que Kant comprend par cette méthode de représentation? Les propositions que Bühler fait à propos des présentations indirectes comme par exemple l'ordre psychique semblent être une manière de répondre à cette question.

Vingt ans plus tard, dans la Krise der Psychologie, Bühler revient à cette discussion et propose un changement de terminologie, en soulignant par-là explicitement la source kantienne de ses réflexions. Il déclare qu'à la place de l'expression d'» ordre psychique », il utilisera maintenant une autre, celle de "schème de représentation"(Darstellungsschema), qui parlerait tout à fait de la même chose :

Il y a 20 ans, j'ai conçu le concept d'» ordre psychique des choses » et je voulais pointer par-là deux choses : d'une part, le fait phénoménologique que dans la pensée actuelle les choses sont souvent seulement indirectement présentes et déterminées, à savoir par la place qu'elles occupent dans un ordre conscient quelconque. Un schème de représentation, comme je l'exprime maintenant d'une manière plus précise, un schème spatial, temporel, syntaxique ou d'une toute autre nature et provenance et, en lui, une place

${ }^{1}$ E. Kant, Critique de la raison pure, 1887, trad. fr. par A. Tremesaygues et B. Pacaud, Paris, 1993, p. 152. Ce paragraphe est cité par Bühler in K. Bühler, «Tatsachen und Probleme zu einer Psychologie der Denkvorgänge: I. Über Gedanken », op. cit., p. 342. Cf. aussi la manière dont Bühler discute cette influence de Kant sur sa propre pensée vingt ans plus tard : K. Bühler, Théorie du langage, op. cit., p. 387.

113 
marquée, une place à laquelle ce qui est pensé appartient, est donnée dans la conscience et présente ce qui est pensé. Dans une pensée à caractère rapide et éphémère, notamment dans la mesure où il s'agit d'objets abstraits, nous opérons très sûrement, efficacement et d'une manière économique avec de telles présentations, qui sont symboliques ou fidèles aux apparences, mais très pauvre en détails. Nous ne pouvons pas seulement relever de manière immédiate dans ces présentations les relations avec d'autres choses du même ordre, mais même au-delà de ce domaine du relevable, nous pouvons encore les prélever de manière juste ${ }^{1}$.

« Substitut vide », « schème », « ordre », «présentation symbolique » désignent donc la même chose : des auxiliaires attestables dans la pensée et sans lesquelles elle s'avère impossible. La thèse qui sera développée dans la dernière partie de cette étude suggère qu'un des leitmotivs de toutes les recherches postérieures à la psychologie de la pensée aura été la description de ces "présentations symboliques» ou "fidèles», comme Bühler les appelle. C'est justement dans la Sprachtheorie que cette idée trouve une large application. Pourtant, comme annoncé, le premier auxiliaire langagier a été déjà découvert dans sa psychologie de la pensée.

\subsection{La découverte du schème syntaxique}

Les expérimentations sur la pensée avaient montré qu'un savoir de la structure grammaticale précédait souvent la formulation de la réponse. Bühler parle aussi d'un vécu particulier qui s'intercale entre le mot et la pensée, et il fait appel à nos propres expériences. Chacun aurait déjà observé qu'en tentant d'exprimer une pensée compliquée, on fait souvent un plan au niveau grammatical. Tout d'abord, c'est la forme de la phrase qui est choisie, et puis la pensée se déploie dans celle-ci. Ou encore, si nous voulons comprendre un texte compliqué, c'est un savoir de la structure grammaticale qui nous guide pour y arriver. Bühler utilise alors l'expression «schème syntaxique » pour discuter ces phénomènes vécus et observés. La description qu'il donne de ce schème syntaxique ne se distingue guère de ce qu'il a dit sur la conscience de la règle et l'ordre psychique des choses. Résumons-le rapidement. 1/ Le schème syntaxique est un savoir, "en l'occurrence un savoir quant à la forme phrastique et quant à la relation des constituants

${ }^{1}$ K. Bühler, Krise der Psychologie, op. cit., p. 116. 
phrastiques entre eux $»^{1}$, présent dans le déploiement de la parole. $2 / \mathrm{Ce}$ savoir constitue un fait phénoménologique. Le schème syntaxique est «quelque chose qu'il faut considérer comme une expression directe des règles grammaticales qui sont vivantes en nous $»^{2}$, dit Bühler. Comme il l'a postulé pour la conscience de la règle, il ne s'agit nullement d'un savoir que de la grammaire. 3/ Il existe une relative indépendance entre ce schème syntaxique et le contenu de l'expression langagière. Les deux peuvent emprunter des voies séparées et en conséquence être appréhendés séparément par le locuteur, mais aussi, rétrospectivement, par le psychologue ou le linguiste. 4/ Cette relative indépendance témoigne du fait que ces schèmes syntaxiques sont vides (ou partiellement vides). Ici, la comparaison avec les fonctions mathématiques s'impose. Comme ces dernières, ils peuvent être pensés en tant que tels sans contenu de remplissement. 5/ Le schème syntaxique joue le rôle de guidage pour la parole effective, il oriente la parole qui se déploie. Voici comment Bühler le décrit :

Lorsque nous débutons une incise par «quand» et qu'à la fin de la subordonnée nous nous interrompons brusquement; nous prenons alors conscience que nous avons attendu quelque chose ; il ne s'agit pas seulement d'un complément matériel, mais aussi d'un complément grammatical, nous attendons une proposition principale. Dans tous ces cas, nous prenons conscience sous forme séparée de ce qui, incidemment et sans qu'on y prête spécialement attention, sert toujours ou presque toujours de médiateur entre les pensées et les mots ${ }^{3}$.

Dans son habilitation, il cite des exemples tirés des procès-verbaux d'expérimentation et propose au lecteur de vérifier ses conclusions à partir de ces données empiriques. Ainsi, il écrit: «Et nombreuses étaient alors les descriptions exposant comment un schème syntaxique totalement ou partiellement vide précédait la formulation proprement dite d'une réponse, et on pouvait reconnaître d'une manière ou d'une autre qu'il guidait la parole effective $»^{4}$. Ce qui étonne toutefois, quand on lit ce que les participants rapportent de leurs vécus, c'est l'utilisation de termes techniques, de termes

${ }^{1}$ K. Bühler, «Tatsachen und Probleme zu einer Psychologie der Denkvorgänge: III. Über Gedankenerinnerungen », op. cit., p. 86.

${ }^{2}$ Ibid., p. 86, voir aussi K. Bühler, Théorie du langage, op. cit., p. 389.

${ }^{3}$ K. Bühler, « Tatsachen und Probleme zu einer Psychologie der Denkvorgänge: III. Über Gedankenerinnerungen », op. cit., p. 86.

${ }^{4}$ K. Bühler, Théorie du langage, op. cit., p. 389. 
qui semblent plutôt être des outils d'interprétation que des termes descriptifs. Prenons quelques exemples :

Les mots venaient sous le guidage de la conscience de la forme phrastique. $(\text { K.E44) })^{1}$

J'avais au début quelque chose comme un filet, dans lequel les mots devaient se faire prendre. $($ S.E51)

J'avais, avant que les mots soient venus, une conscience du rythme, quelque chose comme un schème temporel. (Pf.E31)

Avant les mots, j'avais la conscience d'une forme, quelque chose dans laquelle les mots futurs se rangeaient. (D.A201) ${ }^{4}$

On le voit, les termes de « forme » et même de « schème » sont utilisés pour parler du vécu de parole, ce qui s'explique sans aucun doute par le fait que la majorité des participants étaient des collègues de Bühler. Il est fort probable que ces termes «circulaient» dans les discussions communes de l'équipe et que leur emploi allait ainsi de soi. Le problème auquel est confronté tout interprète de données de ce genre est clair : le choix des mots pour parler de ce que l'on a vécu donne déjà à l'interprétation une certaine orientation. Mais l'inverse est également vrai : des interprétations déjà existantes peuvent influencer la manière dont les participants relatent leur vécu. On est donc moins confronté à une distorsion des résultats qu'à ce fameux «cercle herméneutique » caractéristique de la majorité des recherches en sciences sociales et humaines ${ }^{5}$.

Il reste que, selon l'hypothèse de Bühler, le schème syntaxique est » bel et bien présent quand nous parlons », comme c'est le cas pour la conscience de la règle ou pour l'ordre psychique des choses quand nous pensons. Et même si nous n'arrivons pas toujours à bien le cerner, il

\footnotetext{
${ }^{1}$ K.Bühler, « Tatsachen und Probleme zu einer Psychologie der Denkvorgänge: III. Über Gedankenerinnerungen », op. cit., p. 86.

${ }^{2}$ Ibid.

${ }^{3}$ Ibid., p. 87.

${ }^{4}$ Ibid.

${ }^{5}$ Cf. la description fort pertinente que Taylor donne de ce cercle: Ch. Taylor, «L'interprétation et les sciences de l'homme», in Ch. Taylor, La liberté des modernes, Paris, PUF, 1997, p. 137-142.
} 
constitue une condition nécessaire du fonctionnement de nos langues, qui doit être rendue visible et vérifié :

Ordinairement ce schème syntaxique n'est pas vide, quoique bien présent. Quant à la question de savoir si nous éprouvons ce schème comme isolable ou non, dans la pratique elle est secondaire. Si la technique d'observation de l'époque ne suffit pas, il faut trouver de nouvelles voies pour aller plus loin. Il ne faut pas que les résultats obtenus dépendent définitivement de la subtilité sophistiquée avec laquelle nous décrivons les expériences que nous faisons de notre propre pensée, il faut s'efforcer de les rendre également accessibles à des regards moins subtils, et il s'agit plus encore de les vérifier objectivement ${ }^{1}$.

D'ailleurs, notre auteur change encore une fois d'expression pour désigner ce type de faits. J'ai déjà indiqué que Bühler abandonne l'expression un peu métaphorique d' " ordre psychique des choses" pour celle de "schèmes de représentation ». Dans la Sprachtheorie, le terme de "schème» va être remplacé peu à peu par celui de «médiateur ». Ce dernier terme exprime un concept-clé et prend une place centrale dans cet ouvrage, ce qui se manifeste notamment par le fait que la seule et unique définition du langage qu'on y trouvera se base sur lui : "L'instrument représentationnel langagier fait partie des moyens de représentation indirects ; c'est un instrument intermédiaire (mediales Gerät), dans lequel des médiateurs (Mittler) déterminés interviennent comme facteurs organisateurs $»^{2}$. C'est aussi dans la Sprachtheorie que Bühler insiste encore une fois sur l'origine du concept de médiateur et signale son lien intime avec le concept kantien de schème :

Le concept central de champ symbolique du langage est animé et porté par une idée directrice dont les prémisses sont liées en ce qui me concerne à des études sur la Critique de la raison pure de Kant. En plusieurs endroits de son système, Kant introduit explicitement un médiateur, et ce médiateur est régulièrement caractérisé et décrit comme un schème ${ }^{3}$.

J'insiste quelque peu sur ces références explicites à Kant, puisque la présentation de l'école brentanienne comme anti-kantienne est parfois trop

\footnotetext{
${ }^{1}$ K. Bühler, Théorie du langage, op. cit., p. 390

${ }^{2}$ Ibid., p. 262.

${ }^{3}$ Ibid., p. 387.
} 
vite élargie à la pensée de Bühler ${ }^{1}$. Mais une autre omission dans les études sur Bühler me semble plus importante encore. Il s'agit de l'ignorance quant à la continuité incontestable entre ses réflexions sur le langage publiées en 1934 et ses recherches antérieures sur la psychologie de la pensée. Certainement, toute œuvre classique peut être exploitée pour fournir des réponses ou apporter des idées aux débats ayant lieu à un moment donné. Or, vu la richesse de la pensée bühlerienne sur le langage, il n'est pas étonnant que cet auteur est redécouvert dans les années 70 comme père du tournant sémiotique et dans les années 80 comme précurseur du tournant pragmatique en sciences du langage. Pourtant, si on lit les analyses et les réflexions théoriques que Bühler propose dans la Sprachtheorie comme une continuation de ses recherches sur la pensée, c'est l'idée de ces fameux médiateurs, de ces auxiliaires qui se présente comme centrale pour sa conception du langage. J'ai parlé dans un autre texte du "pouvoir du langage» afin de signaler que les locuteurs et auditeurs, qu'ils le veuillent ou non, sont inévitablement contraints de se servir de ces médiateurs et de se faire guider par eux, car le fonctionnement du langage l'impose ${ }^{2}$. Dans le déploiement hic et nunc de la pensée et de la parole, ces médiateurs (conscience de la règle, schèmes de représentations, schèmes syntaxiques) sont présents et indispensables.

\section{Conclusion}

Peut-on montrer un lien entre l'idée husserlienne de grammaire pure et la thèse de l'existence de schèmes syntaxiques telle que développée par Bühler dans sa psychologie de la pensée et plus tard dans la Sprachtheorie? Que ces médiateurs qui guident indiquent en même temps l'existence de lois logiques ou grammaticales qui, elles, ont un caractère objectif, Bühler l'admet, nous l'avons vu, notamment dans son habilitation. D'ailleurs, le schème syntaxique pourrait être interprété comme ce qui impose au locuteur de suivre, dans le déploiement de la parole, cette loi a priori de placement des parties dans le tout dont parle Husserl. Dans la Sprachtheorie, Bühler reprend cette question de l'objectivité, en montrant que le schème syntaxique des langues

${ }^{1}$ Fiorenza Toccafondi soutient aussi, dans son étude sur Bühler, que « le binôme Bühler-kantisme est entièrement défendable » (F. Toccafondi, «De Karl Bühler à Karl R. Popper », Philosophiques, 1999, t. XXVI, n² 2, p. 293).

${ }^{2}$ J. Friedrich, «Karl Bühler : une pensée du langage », Verbum, tome XXXI, n ${ }^{\circ}-2$, 2009, 3-26. 
indoeuropéennes est typiquement un schème d'action, qui nous oblige à parler du monde presqu'exclusivement sous forme d'action. Il rejoint ainsi Humboldt, qui voit dans ce fait la preuve que les langues indoeuropéennes expriment une conception bien spécifique du monde. En passant de Husserl à Humboldt, Bühler y reconfirme la référence des médiateurs à une objectivité, même si cette objectivité des lois grammaticales devient, dans la lecture qu'il fait de Humboldt, relative à un type de culture.

Toutefois, ce qui intéresse Bühler, ce n'est pas la nature objective ou $a$ priori de ces lois, mais les médiateurs bien spécifiques qui assument la «traduction » ou plus précisément l'action de ces lois dans le fonctionnement psychique. Ce qui anime la démarche de Bühler diffère de ce qui mène Husserl à s'intéresser à une grammaire pure. Les raisons de Bühler ne sont pas épistémologiques, mais psychologiques. Le point essentiel est que Bühler ne cherche pas à déceler les lois (empiriques ou aprioriques) de la pensée, mais à découvrir les «leviers des lois », à savoir ce qui fait que ces lois organisent l'acte de pensée ou l'acte de parler, ce qui fait que l'on pense adéquatement la fonction mathématique ou que l'on parle des choses du monde comme si elles étaient des sujets d'action. Pour Bühler, il n'y a aucun doute que ces médiateurs ont un statut réel ou matériel dans le fonctionnement du psychisme. Il nous invite, dans la Sprachtheorie, à nous efforcer de les rendre accessibles au regard, à les vérifier objectivement. Son intervention porte sur cette question, car la nature psychologique de la pensée et de la parole impose que ces processus soient réalisés indirectement. Il ne faut pas oublier que le contenu des actes de penser et des actes de parler, n'étant pas composé de représentations ni d'images mentales, ne peut être constitué qu'indirectement, et que pour cela, l'appareil psychique se sert de médiateurs. On peut résumer ces différents éléments en utilisant le schéma suivant :

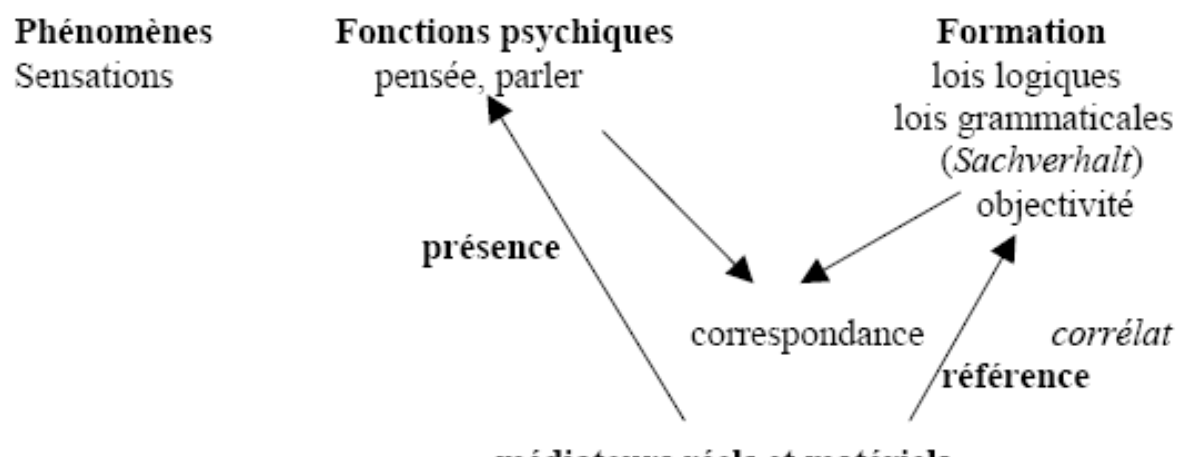

médiateurs réels et matériels

119 
Cette illustration montre deux choses. Premièrement, on voit qu'il y a une correspondance entre le contenu des fonctions psychiques et les corrélats ou formations. La deuxième est plus intéressante pour le chercheur en sciences empiriques. Bühler affirme que les médiateurs présents dans la pensée et dans le parler sont réels et matériels. L'objectif des linguistes et des psychologues serait dès lors de les rendre visibles, de vérifier objectivement leur présence phénoménologique, de les saisir en tant que ce qu'ils sont, à savoir comme constituants vécus du fonctionnement psychique. D'ailleurs, on peut lire la Sprachtheorie comme une proposition d'analyse fine des médiateurs langagiers. Sous un tel angle, c'est bel et bien la discussion des organisateurs déictiques (auxiliaires sensibles) et des organisateurs contextuels (les auxiliaires matériels d'organisation et les auxiliaires syntaxiques) qui retiendront l'attention du lecteur ${ }^{1}$. L'affirmation de Bühler que l'objet de ces médiateurs sont les formations montre en revanche qu'il s'oppose à toute tentative de présenter le contenu de la pensée et de la parole comme produit d'un acte individuel, autrement dit comme composé par des images mentales ou par des représentions, donc psychologiquement.

Peut-on dès lors dire que les deux projets, celui de la grammaire pure de Husserl et celui de l'analyse de la langue comme "mediales Gerät» défendu par Bühler, se rejoignent ? L'objectif déclaré de Husserl a été de fonder sur la grammaire pure une épistémologie en avertissant en même temps les linguistes de l'existence de lois grammaticales a priori. En fait, ce n'est pas tellement cette hypothèse d'une distinction entre lois empiriques et lois aprioriques qui se retrouve chez Bühler quand il discute la conscience de la règle ou le schème syntaxique, mais ce qui l'intéresse, c'est ce qui permet aux lois aprioriques de «prendre corps » dans le déploiement de la pensée et dans le déploiement de la parole. Ces lois a priori sont agissantes au sein du fonctionnement psychique sous forme de médiateurs qui guident et orientent, et qui, selon Bühler, restent à rechercher et à décrire objectivement.

${ }^{1}$ Voir aussi J. Friedrich, «Présentation », in K. Bühler, Théorie du langage, op. cit., p. 21-58. 